\title{
Design of Hand Gesture Controlled Robot using Arduino Lilypad
}

\author{
Balaji Sivakumar, Pravin kumar, Bhuvaneswari Balachander
}

\begin{abstract}
This paper proposes a style of hand gesture controlled automaton victimization Arduino Lilypad. The model projected is controlled through a motion device that is mounted on the hand gloves. This style helps physically challenged folks and additionally for sure tasks educated by human. the most aim of this style is to manage the automaton victimization hand gesture. measuring device utilized in the planning senses the direction of hand movement and sends an indication to Arduino Lilypad. Four main Hand gesture movements like FORWORD, BACKWORD, LEFT and RIGHT area unit detected and enforced.
\end{abstract}

Keywords: Arduino Lilypad, Accelerometer, hand gesture, wireless robot, physically challenged.

\section{INTRODUCTION}

People would like to regulate everything with my hands! Sitting in my chair and dominant things sort of a BOSS. thus I finally came out with a hand gesture recognition automaton, which might follow the commands created by hand gestures. Sounds crazy however I promise it's terribly easy. The automaton is split into 2 necessary elements, transmitter and receiver. Here we'd like to program the transmitter circuit. thus i will be able to be mistreatment AN Arduino because the programming platform. To sense the gestures created $\mathrm{i}$ will be able to be mistreatment AN measuring system detector. Let's see deep part operation of style of Hand Gesture Controlled automaton mistreatment Arduino Lilypad and let's see deep operation circuit operating of style of Hand Gesture Controlled automaton mistreatment Arduino Lilypad

\section{Block diagram}

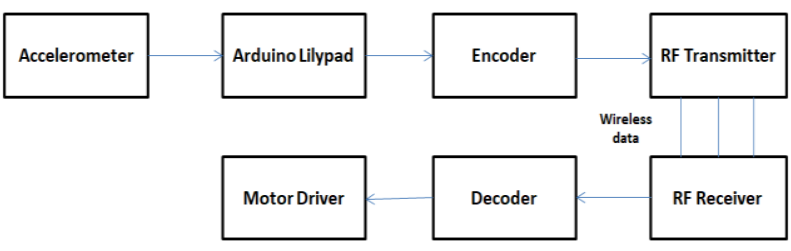

Fig 1.

\section{OPERATION OF COMPONENTS INVOLVED}

Accelerometer ADXL335

Revised Manuscript Received on August 14, 2019.

Balaji Sivakumar, Department of Electronics and Communication Engineering, Saveetha School of Engineering, Thandalam, Chennai, Tamilnadu, India (email: balaji.s.maha@gmail.com)

Pravin kumar, Assistant professor, ECE department, Saveetha School of Engineering,Thandalam, Saveetha University, Chennai, Tamilnadu, India(email: pravintiny@gmail.com)

Bhuvaneswari Balachander,(email: bhuvaneswari@saveetha.com)
An measuring device may be a 3 -axis acceleration activity part. The measuring device that used here is ADXL335 and it's three axes X, Y, Z. the ADXL335 is part that measures the vibration or motion of structure the force of vibration or a amendment in motion this affects the 'squeeze' the electricity material which provides AN electrical charge that's proportional to force exerted upon it .the charge is proportional to the ADXL335.

Now days all good phones have measuring device that used for motion games for e.g. temple run.

An measuring device has half-dozen pins that as VCC, GND, X, Y and Z and conjointly ST. Vcc is connected to the $5 \mathrm{v}$ power offer. $\mathrm{X}$ and $\mathrm{Y}$ area unit connected to the Arduino Lilypad pins of 7(A0) and 8(A1)

\section{Microcontroller Arduino Lilypad}

Arduino Uno is that the microcontroller board supported the ATmega328p, Arduino Uno it's the center of the project that planning to management the golem by giving the directions and there ar many varieties of Arduino boards however here the sort of board is Arduino Lilypad, even this additionally planning to do an equivalent method however with some distinction, the distinction that is nothing however system voltage, clock speed, digital I/O, analog inputs and programming interface and additionally the price.

\section{Encoder HT12E \& Decoder HT12D}

Encoder HT12E and Decoder HT12D, the 12 which means 8 address lines and 4 information lines and $\mathrm{E}$ and $\mathrm{D}$ letter speaks to the Encoder and Decoder Crafted by HT12E and HT12D which dependent on 8 address lines and 4 information lines ,according to information sheet the information going to HT12E "4 information lines" which wrapping that info information and goes to the HT12D , HT12D translates the wrapped information by Encoder

The HT12E arrangement encoder is utilized. It is equipped for encoding data that comprises of $\mathrm{N}$ address bits? It has 18 pins. Stick ( 1 to 9 ) and 14 are associated with ground. Stick number 10,11,12,13 of the encoder are associated with 8, 9, 10, and 11 of Arduino Lilypad board. A resistor (10kohm) is associated between sixteenth stick and fifteenth stick. seventeenth stick connected (associated) to Data stick of $433 \mathrm{MHz}$ RF transmitter module. IC deals with $5 \mathrm{v}$ power supply, VDD is associated with stick eighteenth

The HT12D arrangement decoder is utilized. It is

\section{Published By:}

Blue Eyes Intelligence Engineering \& Sciences Publication 
equipped for interpreting data that comprises of $\mathrm{N}$ address bits. It has 18 pins. Stick (1 to 9) associated with ground. $10,11,12,13$ pins of decoder are (connected) associated with $10,15,7$, and 2 of Motor drive. A resistor of $47 \mathrm{KOhm}$ is associated between 15 and 16 pins. fourteenth stick is associated with RX433 information stick. IC takes a shot at $5 \mathrm{v}$ power supply, VDD is associated with stick eighteenth.

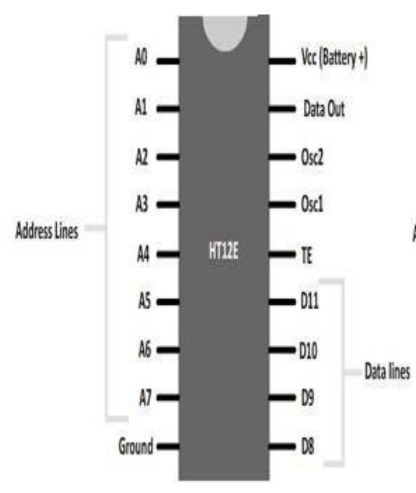

Fig 2.

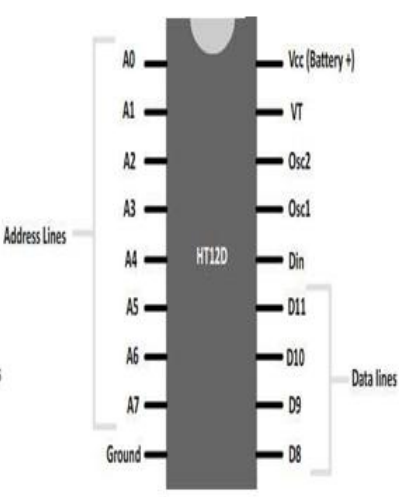

Fig 3.

\section{$R F$ transmitter \& $R F$ receiver:}

RF it means radio recurrence. We have utilized $433 \mathrm{MHz}$ RF Transmitter/Receiver module. It has been planned in various working frequencies and furthermore in various working reach. To transmit and get the sign by means of RF module is important to have an encoder and decoder module which encodes and unravels the sign to transmit and get the sign. The transmitting extent is up to $500 \mathrm{ft}$ with range rate of $1 \mathrm{kbps}$ to $10 \mathrm{kbps}$.

TX module comprises (GROUND, VCC, DATA, ANTENNA) absolutely 4 pins. Encoder seventeenth stick is associated with the information stick of the module. A $17 \mathrm{~cm}$ reception apparatus is utilized to transmit and get the sign which are associated with radio wire stick of TX module. TX gets sequential information and RF sign is transmitted remotely to the collector by means of this radio wire.

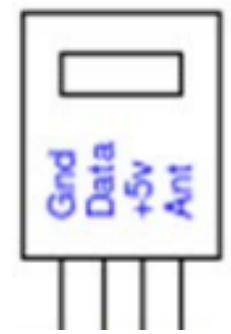

Fig 4.

RX module comprises 3 ground pins, 2 VCC pins, 2 DATA pins and 1 radio wire stick absolutely 8 pins. Decoder fourteenth stick is associated with the information stick of the Rx module is. A $17 \mathrm{~cm}$ recieving wire is utilized to transmit and get the sign which are associated with radio wire stick of RX module. RF sign is transmitted remotely to the recipient by means of this recieving wire.

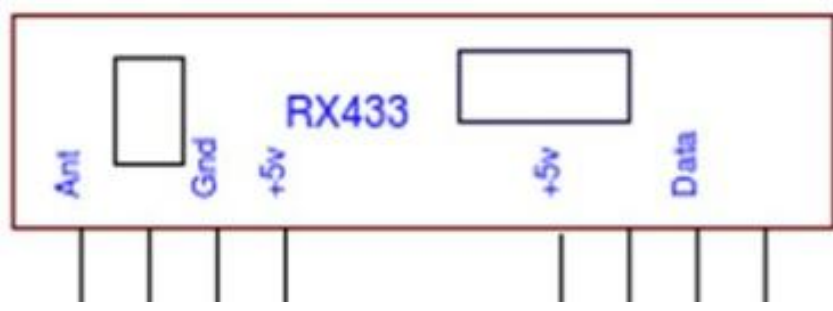

Fig 5.

The reception apparatus length can be dictated by scope of RF module recurrence.

We know:

Wavelength $(\lambda)=$ speed of light (c) / frequency (f)

$\lambda=$ wavelength, the separation between wave peaks $(\mathrm{m})$

$\mathrm{c}=$ speed of light $(\mathrm{c})$

$\mathrm{f}=$ recurrence, the wave peaks that experience a point in a specific time (cycles/s or $\mathrm{Hz}$ )

$=3 \times 10 / 433 \times 10 \mathrm{~m}$

$=0.69284 \mathrm{~m}$

So; $\quad$ antenna length $=\lambda / 4$

$=0.69284 / 4 \mathrm{~m}$

$=0.1732 \mathrm{~m} \approx 17 \mathrm{~cm}$

\section{Motor driver}

L293D IC which is utilized here and it has 16 stick which has 4 information pins, 4 yield pins, 4 VCC pins and 4 ground pins. Every one of the 4 information pins are associated with decoder IC yield pins. Also, four yield pins are associated with the engines of the robot. Interface each of the 4 VCC pins to 5V DC supply.

\section{RESULTS}

\section{Design and working}

The Transmitter part is kept on palm and the beneficiary part on robot vehicle that moves as indicated by the hand development. This paper depicts about the five distinct sorts of motion places of the hand. This is only 'stop condition', 'forward condition', 'in reverse condition', 'right' and 'left'.

\section{Stop Condition}

At the point when the accelerometer position is even arrangement, every one of the yields of the decoder will be high. That yield pins are 13,12,11 and 10 those are set to 1 (high) which makes robot to stop.

\section{Forward condition}

At the point when the accelerometer position is tilted to advance. Decoder yield pins 13, 11 are set to 0 (low) and other two yields pins of 12,10 are set to 1 (high) which makes robot to push ahead

\section{Backward condition}

At the point when the accelerometer position is tilted to towards in reverse position. Decoder yield pins 12, 10 are set to 0 (low) and other two yields pins of 13,11 are set to 1 (high) which makes robot to move in reverse.

\section{Right condition}

At the point when the accelerometer position is tilted to towards right position. Decoder yield pins 12,11 are set to 0 (low) and other two yields pins of 13, 10 are set to 1 (high) which makes robot to move towards right.

\section{Left condition}

At the point when the accelerometer position is tilted to towards left position. Decoder yield pins 13, 10 are set to 0 (low) and other two yields pins of 12,11 are set to 1 (high) which makes robot to move towards left.

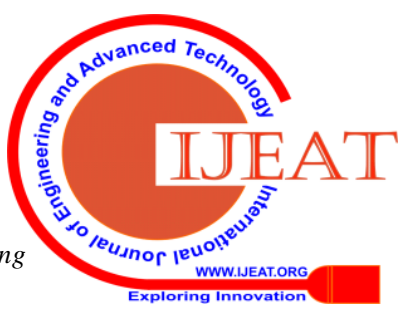




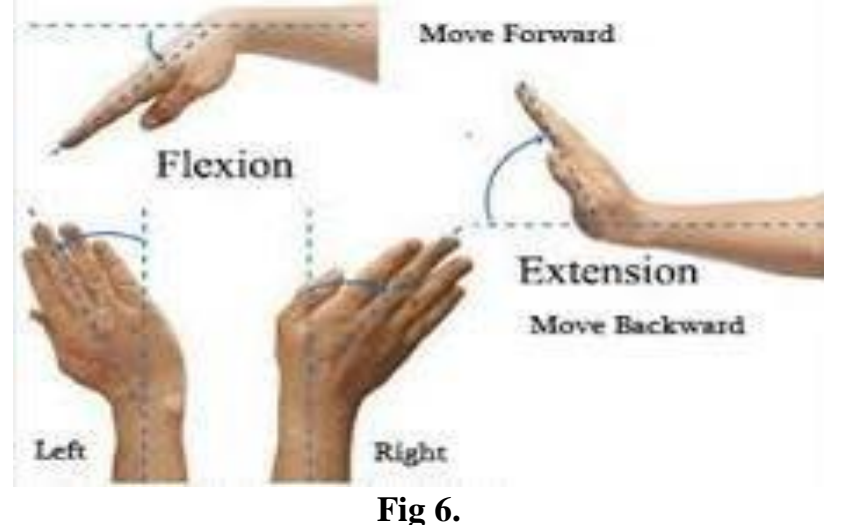

Transmitter circuit for the system

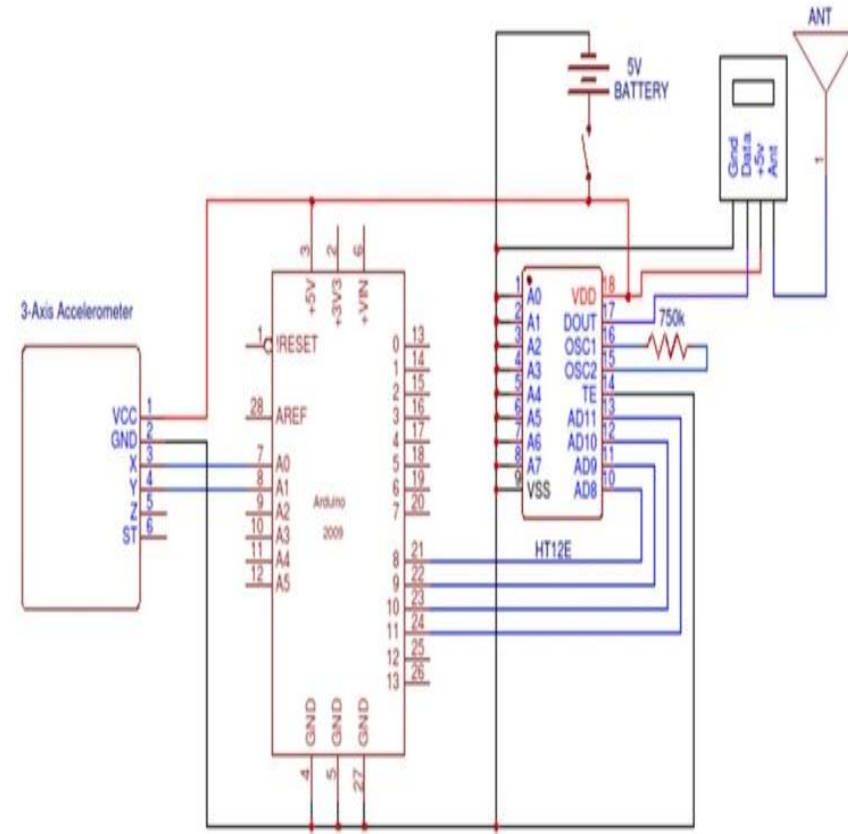

Fig 7: (TX)

Receiver circuit for the system

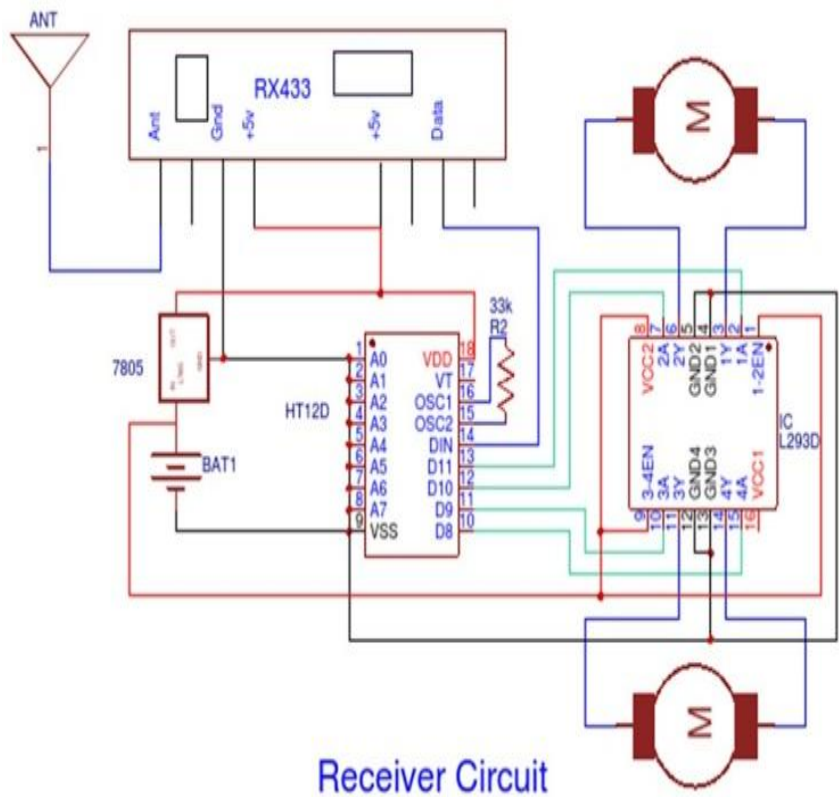

Fig 8: (RX)

\section{Circuit Elements}

The fig 7 comprises of transmitter circuit which has a few of segments included which previously clarified above and fig 8 comprises of beneficiary circuit which has a few of part required to get unique sign of transmitter and RX as of now clarified previously.

\section{Result}

The sign transmission through the radio recurrence segment which has better capacity contrast with IR (infrared) .And primary favorable position of utilizing RF, which can transmit the sign through longer separations this aches extend application, and furthermore sign can travel if there any obstacle between TX and RX. The working recurrence of transmitter and beneficiary is $433 \mathrm{MHz}$ and Radio transmitter recipient through the recieving wire stick. The sequential information is transmitted through radio wire and recipient gets the information and changes over to unique type of sign and move to engine driver and engine driver works the engine according to the client hand movement..

\section{REFERENCES}

1. ACCELEROMETER BASED GESTURE CONTROLLED ROBOT USING ARDUINO SwarnaPrabha Jena, Sworaj Kumar Nayak, Saroj Kumar Sahoo, Sibu Ranjan Sahoo, Saraswata Dash, Sunil Kumar Sahoo

2. Accelerometer Based Hand Gesture Controlled Robot Mr.Pravin Vaishnav1, Mrs.Shalini Tiwari2

3. Hand Motion-Based Remote ControlInterface with Vibrotactile Feedback for Home Robots

4. Hand Gesture Based Wireless Controlled Robot Dushyant Singh, Riturvanjali Singh, Neelam Sharma,Sagar Shrivastav , and Ashutosh Mittal

5. http://www.sensorwiki.org/doku.php/sensors/accelerome ter

6. https://www.edn.com/electronics-blogs/eye-on-iot/4437311/Using-433-MHz-for-wireless-connectivity-inthe-Internet-of-Things

7. https://www.arduino.cc/en/Main/ArduinoBoardLilyPad/ 